\title{
Pharmacologic and surgical therapies for patients with Meniere's disease: a protocol for a systematic review and meta-analysis
}

\author{
Nadera Ahmadzai ${ }^{1}$, Wei Cheng ${ }^{1}$, Dianna Wolfe ${ }^{1}$, Jamie Bonaparte ${ }^{2}$, David Schramm², Elizabeth Fitzpatrick ${ }^{3,4}$, \\ Vincent Lin ${ }^{5,6}$, Becky Skidmore ${ }^{1}$, Leila Esmaeilisaraji ${ }^{1}$, Shaun Kilty ${ }^{1,2,7}$ and Brian Hutton ${ }^{1,8^{*}}$ (D)
}

\begin{abstract}
Background: Hearing loss is one of the leading causes of disability in Canada and worldwide, with more than one million Canadians enduring a hearing-related disability. Meniere's disease (MD) is a chronic condition of the inner ear, manifesting as a triad of disabling symptoms, including attacks of vertigo, fluctuating sensorineural hearing loss (SNHL), and tinnitus. Impacts on quality of life are severe, particularly with respect to restrictions in social participation and physical activity, fatigue, and reduced capacity to work. Anxiety and other psychological disorders may result from the restrictions imposed on life, the constant uncertainty of vertigo attacks, and fluctuating SNHL, with neuroses and depression affecting 40 to $60 \%$ of sufferers of intractable MD. There is a need to establish the benefits of previously studied interventions with greater certainty. The planned systematic review and meta-analyses/network meta-analyses (NMAs) will assess the relative effects of competing pharmacologic and surgical interventions for management of MD in adults.
\end{abstract}

Methods: An experienced medical information specialist in consultation with the review team will develop the electronic search strategies. We will search various databases including MEDLINE, Embase, and the Cochrane Library with no date or language restrictions for published literature, and key clinical trial registries for in-progress and completed trials. Screening of the literature will be performed by two reviewers independently using pre-specified eligibility criteria, and quality of the included studies will be assessed using the Cochrane Risk of Bias tool. We will resolve disagreements through consensus or third-party adjudication. When applicable, meta-analyses and NMAs will be pursued to compare interventions in terms of their effects on outcomes, including frequency and severity of vertigo, occurrence and intensity of tinnitus, changes in hearing and speech recognition, quality of life, and harms. Separate analyses exploring the effects of pharmacologic and surgical approaches will be performed.

Discussion: Our planned systematic review will provide informative evaluations of existing treatments for management of Meniere's disease. The findings will inform practitioners as to the relative benefits and harms of the existing competing interventions for MD, offer optimal clinical treatment strategies, identify evidence gaps, and determine promising therapies for evaluation in future trials.

Systematic review registration: PROSPERO CRD42019119129

Keywords: Meniere's disease, Meta-analysis, Network meta-analysis, Systematic review

\footnotetext{
* Correspondence: bhutton@ohri.ca

${ }^{1}$ Center for Practice Changing Research, Ottawa Hospital Research Institute,

501 Smyth Road, Box 201B, Ottawa, ON K1H 8 L6, Canada

${ }^{8}$ The University of Ottawa Faculty of Epidemiology and Community

Medicine, 451 Smyth Rd, Ottawa, ON K1H 8L1, Canada

Full list of author information is available at the end of the article
}

(c) The Author(s). 2019 Open Access This article is distributed under the terms of the Creative Commons Attribution 4.0 International License (http://creativecommons.org/licenses/by/4.0/), which permits unrestricted use, distribution, and reproduction in any medium, provided you give appropriate credit to the original author(s) and the source, provide a link to the Creative Commons license, and indicate if changes were made. The Creative Commons Public Domain Dedication waiver (http://creativecommons.org/publicdomain/zero/1.0/) applies to the data made available in this article, unless otherwise stated. 


\section{Background}

Hearing loss is one of the leading causes of disability in Canada and worldwide, with more than one million $\mathrm{Ca}-$ nadians enduring a hearing-related disability $[1,2]$. Despite the availability of various interventions, Canadians with hearing loss may endure a diminished quality of life [3]. Meniere's disease (MD) is a condition that frequently causes hearing loss and may have a large emotional and financial toll on patients, their families, and society that is often underestimated [4]. MD has a variable clinical course [5]. For instance, hearing loss may spontaneously increase with a concurrent increase in the associated symptomatology of the disease, including potentially incapacitating vertigo. The spontaneous changes in the symptomatology of MD, including further hearing loss, make treatment particularly difficult when the disease symptoms are continually changing and the changes are not predictable.

$\mathrm{MD}$ is a chronic condition of the inner ear, manifesting as a triad of disabling symptoms, including attacks of vertigo, fluctuating sensorineural hearing loss (SNHL), and tinnitus [6]. Impacts on quality of life can be severe, particularly with respect to restrictions in social participation and physical activity, fatigue, and reduced capacity to work. Anxiety and other psychological disorders may result from these restrictions on life, the constant uncertainty of vertigo attacks, and fluctuating SNHL $[6,7]$, with neuroses and depression affecting 40 to $60 \%$ of sufferers of intractable MD [8]. The symptoms of MD result from an increase in the hydraulic pressure within the inner ear endolymphatic system, termed "endolymphatic hydrops" (EH). Currently, MD diagnosis is symptom based, with guidelines that define diagnostic criteria and certainty [9]. Management of MD focuses on treatment and prevention of vertigo attacks, improvement or preservation of hearing and vestibular function, and prevention of bilateral MD [10]. Both medical and surgical interventions have been used [11]. Pharmaceutical interventions include systemic (e.g., diuretics, antihistamines) as well as intra-tympanic therapies (e.g., gentamicin, steroids) aimed at either reducing pressure in the endolymphatic system or chemical labyrinthectomy $[10,11]$. Dietary therapies to reduce endolymphatic pressure include restrictions of salt, water, alcohol, or caffeine [10]. Surgical treatment involves conservative procedures in which the hearing preservation is attempted or destructive labyrinthectomy in which hearing is lost [10]. Psychological support may improve results of treatment for patients with intractable MD [8].

The detrimental effects of MD on patients' quality of life may be severe, and thus early detection and rapid intervention are encouraged [7]. However, the uncertainty surrounding the efficacy of various available interventions makes the selection of appropriate treatments difficult. This protocol presents methodology for a systematic review incorporating meta-analyses/network metaanalyses (NMAs) that was developed by researchers in collaboration with clinical content experts who prioritized the question of interest.

\section{Objectives}

The primary objective of this systematic review is to assess the relative effects of available pharmacologic therapies in patients with MD on vertigo and other key patient outcomes in randomized controlled trials and quasi-randomized trials. The secondary objective is to assess the effects of surgical interventions in the MD population on vertigo and other key patient outcomes in randomized and quasi-randomized trials.

\section{Methods}

This protocol adheres to the standards of the Preferred Reporting Items for Systematic Review and Meta-Analysis Protocols (PRISMA-P) Statement [12], and is registered with the International Prospective Register of Systematic Reviews (PROSPERO) database (CRD42019119129) [13]. A populated PRISMA-P checklist for this protocol is provided as an additional file (see Additional file 1). Any post hoc modifications to the plans presented within the protocol will be recorded and described in the publication of the final report to ensure transparency. The final report will be developed in consultation with the PRISMA Extension Statement for NMA to ensure all aspects of methods and findings are fully reported [14].

\section{Study eligibility criteria}

We have established the review eligibility criteria based on the PICOS (Population-Intervention-ComparatorsOutcomes-Study design) framework. We will include primary studies that meet the following criteria:

- Population. Studies enrolling adult patients with MD per established criteria (i.e., American Academy of Otolaryngology-Head and Neck Surgery (AAOHNS) [9]) receiving pharmacologic or surgical interventions for their condition (e.g., endolymphatic sac decompression, intra-tympanic gentamicin injection, or others as detailed below) will be sought [15]. In studies with enrollment criteria that required patients to be unresponsive to a prior intervention in order to be treated with the intervention(s) of interest, we will discuss the potential for important clinical heterogeneity with our clinical experts and exercise discretion about whether these studies can be appropriately combined together in quantitative analyses.

- Interventions/comparators. The following interventions will be of interest: 
o Systemic pharmaceuticals: diuretics (e.g., hydrochlorothiazide, furosemide), Motion sickness/anti-nausea medications (e.g., anticholinergics, antihistamines (betahistine), phenothiazines), benzodiazepines (e.g., diazepam);

o Intra-tympanic pharmaceuticals: intra-tympanic gentamicin, intra-tympanic steroids;

- Surgical interventions: sacculotomy, vestibular nerve section, labyrinthectomy, tympanostomy tube, endolymphatic duct blockage, endolymphatic sac decompression, endolymphatic shunt, transtympanic pressure treatment.

While we have structured interventions into broad categories (e.g., systemic and intra-tympanic pharmaceuticals, surgical, and others), our primary analyses will consider interventions at a more granular level to ensure findings are of maximal clinical relevance. We will pursue separate sets of analyses relating to pharmacologic and surgical interventions, as this approach is in alignment with clinical decision-making. After study data has been extracted, we will consult our clinical experts to determine whether additional treatment nodes or other modifications related to network geometry are needed to enhance representativeness of interventions (e.g., considering variable doses/durations of pharmaceutical therapy). If combination therapies are encountered, we will include them as additional treatment groups in the analyses to be performed.

- Outcomes. Endpoints of interest will include the following:

o frequency, severity, type, and control of vertigo measured via electrocochleography score test or other methods;

0 occurrence and intensity of tinnitus measured via various methods such as psychoacoustic tests (pitch match, loudness match, maskability, residual inhibition, etc.), rating scales (e.g., verbal rating scale, numerical rating scale, visual analog scale, poster style, and mechanical device), questionnaires describing functional effects (e.g., tinnitus questionnaire, tinnitus handicap questionnaire, tinnitus severity scale, subjective tinnitus severity scale/tinnitus reaction questionnaire, tinnitus severity grading, tinnitus severity index, tinnitus handicap inventory, intake interview for tinnitus retraining therapy), and patients' global perception of treatment-related changes;

0 changes in hearing, based on Pure Tone Average (PTA) in decibels, and speech recognition, such as word recognition score (WRS) that may also be labeled as speech discrimination score (SDS), and speech reception threshold (SRT);

- quality of life measured by various scales such as Quality of Well-being Scale (QWB), SF-12, Physical SF-12 score; Mental SF-12 score, Center for Epidemiologic Studies-Depression Scale (CESD);

o perception of aural fullness;

$\circ$ and harms, including hearing loss, withdrawals due to adverse effects, and serious side effects defined by authors.

- Study design. Randomized controlled trials and quasi-randomized trials

- Timing. Studies with a minimum follow-up duration of 6 months after the first intervention

- Language. English language

- Setting. Any setting

\section{Information sources and search strategy}

The search strategies will be developed and tested through an iterative process by an experienced medical information specialist in consultation with the review team. The strategies will be peer reviewed by another senior information specialist prior to execution using the PRESS Checklist [16] Using the OVID platform, we will search Ovid MEDLINE ${ }^{\bullet}$, including Epub Ahead of Print and In-Process \& Other Non-Indexed Citations, and Embase Classic+Embase. We will also search the Cochrane Library on Wiley.

Strategies will utilize a combination of controlled vocabulary (e.g., "Meniere Disease," "Endolymphatic Hydrops") and keywords (e.g., "auditory vertigo," "endolymphatic hydrops," "Meniere's"). Results will be filtered using headings for systematic reviews, randomized controlled trials, and non-randomized controlled trials as applicable for each database. Vocabulary and syntax will be adjusted across databases. There will be no language or date restrictions on any of the searches, but when possible, animal-only and opinion pieces will be removed from the results. Potentially relevant articles published in languages other than English will be included in an Additional file. We will document any unforeseen limitations pertaining to search strategy upon its completion.

A gray literature search of targeted clinical trial registries, ClinicalTrials.gov, and the International Clinical Trials Registry Platform will also be undertaken. Studies included in existing reviews will be inspected to confirm no relevant studies have been missed. Content experts will be contacted to obtain information on unknown or ongoing studies. The proposed database search strategies are provided in Additional file 3. 


\section{Screening and data extraction}

Screening will be performed in two stages via two reviewers working independently and in duplicate against eligibility criteria established a priori, using an online systematic review software program (Distiller Systematic Review (DSR) Software; Evidence Partners Inc, Ottawa, Canada). Stage 1 screening will be based on review of the abstracts and titles identified from the electronic search, while stage 2 screening will consider full-text review of the articles deemed potentially relevant during stage 1 . Screening at both stages will commence with a calibration exercise to ensure consistent application of eligibility criteria. A screening pilot will be performed prior to full screening of titles and abstracts (25 titles and abstracts) and full-text screening (25 studies). At stage 1, two reviewers (NA and LE) will independently assess the titles and abstracts for eligible studies using the liberal accelerated method [17] where only one reviewer is required to include citations for further assessment at full-text screening and two reviewers are needed to exclude a citation. At stage 2, the full-text articles of potentially relevant citations will be retrieved for fulltext screening and the same two reviewers (NA and LE) will independently assess the article for relevancy. Disagreements between reviewers will be resolved via consensus or third-party adjudication. The study selection process will be reported using a PRISMA flow diagram [18] in the final publication. References of all included studies will be scanned for inclusion, by one reviewer. At least one content expert will be consulted for additional studies. Study authors will be consulted where necessary for verifying eligibility and for missing or unclear information on studies (and information will be included if received in a timely manner). A list of the excluded studies alongside the rationale for their exclusion will be provided in an additional file to the completed review. With regard to duplicate publications, companion documents, or multiple reports of a primary study, we will collate all available data and use the most complete set and exclude the duplicate version or companion documents with no additional data.

A standardized data extraction form in Microsoft Excel (Microsoft Corporation, Seattle, WA, USA) will be used for collecting key study information that includes all pre-specified data items (see Additional file 2). After piloting the data extraction form on a small number of studies, two reviewers (NA and LE) will extract the data independently and any discrepancies will be resolved by discussion or a third person. Information from each study will be recorded that will include (but not be limited to) the following: publication characteristics (e.g., authors' names, publication year, and journal), study design traits (cited trial design, clinical setting, duration of follow-up, number of patients randomized and number analyzed for each outcome, occurrence of dropouts, funding source, and authors' conflict of interest etc.), study population details (patient inclusion and exclusion criteria, age, sex, body mass index (BMI), race, comorbidities, prior treatments, and relevant baseline data, such as initial PTA, WRS/SDS, hearing loss in the opposite ear previously affected with MD, aural fullness in the opposite ear, time from onset to treatment, prior otologic surgery, presence of tinnitus and vertigo, etc.), intervention and comparator specifics (type, dose, unit, duration, frequency, route of administration, strategy of administration, and co-intervention, etc.), and outcome data (including reported outcome definitions and summary data related to treatment effects (e.g., mean change and the corresponding standard error for continuous outcomes, and number of events and number of total patients for dichotomous outcomes), and reported scales for evaluating the outcomes). A complete list of prespecified data items is presented in Additional file 2. Means and measures of dispersion will be approximated from figures in the primary studies using online tools. When available, data from both intention to treat and per protocol analyses will be extracted. We will contact authors for any missing or additional data of interest. Authors' defined pre-specified outcomes of interest will be extracted and grouped accordingly for analyses.

\section{Outcomes and prioritization}

We explored the Core Outcome Measures in Effectiveness Trials (COMET) initiative [19] but did not locate a core outcome set for Meniere's disease [20]. As such, the endpoints of interest for this review were selected via consultation with our clinical experts. The primary outcome of interest will be vertigo, while the secondary outcomes of interest will include changes in hearing, tinnitus, quality of life, aural fullness, and harms. With regard to outcomes definitions, we will gather outcomes with any definitions provided by the primary study authors. We will group together the data with similar definitions across the studies. Priority will be given to established definitions where possible. For instance, for vertigo control, we will consider the definition from the American Academy of Otolaryngology-Head and Neck Surgery (AAO-HNS) [9]. However, in the event of insufficient outcome data using this definition, we will consider other definitions in consultation with our clinical context experts, and the set with the most available data and clinical relevance will be given priority in analysis. In terms of time of assessment for the endpoints of interest, we will consider data reported according to the AAO-HNS recommendation on reporting the treatment results [9] when available. For instance, for frequency of definitive attacks, outcome data from the period 6 months before treatment should be compared with 
interval occurring between 18 and 24 months posttreatment. However, if insufficient data are reported based on AAO-HNS criteria across primary studies, we will collect outcome data at various time points such as baseline, 6 months, 12 months, and 24 months posttreatment if possible. In the absence of sufficient data, we may consider other reported time points in consultation with our clinical content experts to ensure clinical relevance.

\section{Risk of bias assessment}

We will use the Cochrane Risk of Bias Tool for RCTs [21] to evaluate the risk of bias of each included RCT and quasi-randomized trial. Two reviewers will carry out assessments independently and resolve disagreements via consensus or third-party adjudication. All domains of the Cochrane Risk of Bias tool for RCTs will be considered, including selection bias (sequence generation and allocation sequence concealment), performance bias (blinding of participants and personnel), detection bias (blinding of outcome assessment), attrition bias (incomplete outcome data), reporting bias (selective reporting), and other biases considered relevant to the review topic. We will also evaluate baseline imbalances between groups with respect to comorbidities and factors that may impact our outcomes of interest, including history of falls more than once in the past year, older age, white race, female sex, higher BMI, current tinnitus, prior therapies received, allergies, immune dysfunction (e.g., ankylosing spondylitis, systemic lupus erythematosus, psoriasis), autonomic dysfunction, poor mental health $[18,20,22]$, arthritis [23], history of hearing loss or episodic vertigo [24], familial history of MD, and diabetes mellitus [25].

\section{Approaches to evidence syntheses}

\section{A. Criteria for quantitative synthesis}

To assess the assumptions for conducting NMA (i.e., the transitivity assumption, relating to similarity amongst studies in an evidence network), a variety of information related to study methods, patient demographics, and eligibility criteria will be collected. These will include the following: mean age at onset, percent female patients, mean disease duration, frequency/severity measures of vertigo at baseline, percent with history of migraine headaches, percent with previously identified tinnitus, measures of average tinnitus intensity, and other factors. These study features will be reviewed with our clinical experts using a combination of table summaries, box plots, and bar plots to identify potential outlier studies that may warrant exclusion from formal analyses.
As noted earlier, separate sets of analyses pertaining to comparison of pharmacologic interventions and surgical interventions will be performed. Initially, we will inspect the characteristics of included studies such as patients' clinical characteristics (age, sex, and clinical history, including duration of hearing impairment and baseline severity) and methodologic homogeneity (e.g., risk of bias, study design), and we will summarize them accordingly. A pairwise meta-analysis for each intervention comparison will be pursued to explore statistical heterogeneity (based upon the $I^{2}$ statistic) if data permit.

\section{B. Planned quantitative analysis}

If data permits, both fixed effects and random effects Bayesian NMAs will be performed to compare interventions contained within the included studies that are sufficiently connected for a specified clinical endpoint. A common between-trial standard deviation will be used for Bayesian NMAs as per established methods [26-28]. Model fit will be assessed by comparing total residual deviance with the number of unconstrained data points [29] and will be considered adequate if these quantities are approximately equal. The deviance information criteria (DIC) will be used for selection between models, with a difference of five points suggesting an important difference [29] (with smaller values being preferred). The type of endpoint under analysis (e.g., continuous or binary) will determine the use of specific NMA models. We will use mean change per arm (between pre- and postintervention) for the analysis of continuous endpoints measured in the same units, and the corresponding effect size will be the mean difference. It is common in general that studies report findings of the same continuous endpoint in different formats, some reporting mean changes with corresponding standard errors (SEs), while others report only mean values at baseline and posttreatment with corresponding standard deviations (SDs) for each treatment arm. For the latter scenario, we will consider the appropriateness of assuming a correlation between mean values at baseline and follow-up and calculate the mean changes and corresponding SEs when they are not reported. If we encounter continuous endpoints that are measured using different scales across studies (e.g., a visual analog scale from 0 to 100 versus an itemized, composite score scale to assess severity of vertigo attacks), a model for estimating the effect size as a standardized mean difference (SMD) will be considered to explore benefits across related scales and maximize usage of available data. Estimates of effect sizes for binary endpoints will be expressed as odds ratios. All pairwise comparisons between interventions will be expressed with $95 \%$ credible intervals. Key secondary measures of effect, such as the surface under the 
cumulative ranking curve (SUCRA) and average treatment rankings [30], will be estimated to explore potential orderings of treatments. All NMAs will be carried out using OpenBUGS version 3.2.3 [31] and the R2OpenBUGS package [32] version 3.2-3.2 in $\mathrm{R}$ [31].

We will rely on pairwise meta-analyses if the evidence networks of pharmacologic interventions or surgical interventions are not well-connected. Whenever data permit for each pairwise comparison, we will use funnel plots to assess for small-study effects as a signal of publication bias. Where protocols are available, they will be reviewed to inform evaluations for selective reporting within the set of included studies.

\section{Proposed additional analyses}

If feasible (based upon quantity of available evidence and rigor or reporting of the included studies), we will explore subgroup analyses $[27,33]$ to evaluate robustness of our findings and the impact of covariates. In consultation with our clinical experts, the subgroups will be chosen and may include (but not be limited to) gender distribution (e.g., percent females), age (older versus younger), BMI (higher BMI versus lower BMI), race (white versus others), presence of dizziness, number of days since initial treatment (or onset of MD), severity of initial hearing loss, type of MD (unilateral versus bilateral), and types of unilateral MD [34]: (1) classic MD (sporadic MD, without migraine and autoimmune disorder), (2) delayed MD (hearing loss antedates vertigo episodes for months or years and is without migraine or autoimmune disorder in most cases), (3) familial MD, (4) MD with presence of migraine, and (5) MD comorbid with autoimmune disorder.

\section{Narrative summary}

If excessive heterogeneity is identified and the research team feels meta-analysis is inappropriate, a narrative summary of findings with supporting tables and figures will be prepared.

\section{Discussion}

To date, there have been a variety of pharmaceuticals used for the treatment of MD, including systemic drugs and intra-tympanic medications as well as surgical approaches. There exist systematic reviews comparing effectiveness of pairs of interventions in patients with MD [35-38]. However, these systematic reviews focus only on direct pairwise comparisons (e.g., one intervention versus placebo or another active treatment) [39-41]. When applicable, NMA compares multiple interventions in one analysis and borrows strength from both direct and indirect evidence. Our planned systematic review will employ sound methodology and will provide new and informative evaluations of present competing therapies and their relative benefits in managing MD. As interventions for MD are often chosen using a staged approach, we have planned to focus our review upon separate comparisons of two important aspects of treatment which typically follow insufficient response to lifestyle modifications: pharmacologic interventions and surgical interventions. An important strength of this systematic review (if sufficient data and homogeneity are present) will be the performance of NMA of therapies for the management of MD; to our awareness, this will represent the first such analysis. A second strength will be the assessment of benefits and harms of not only surgical interventions, but also pharmaceutical agents in treating this condition. A potential challenge will be threats to clinical homogeneity across studies including variability in prior treatments received and other relevant factors.

We will publish the findings of our review in a clinical specialty journal to enhance outreach to clinicians pursuing prospective research on MD. We will report evidence networks collating completed studies as well as ongoing trials identified from www.clinicaltrials.gov and other registries to establish the current state of the evidence. In keeping with the COMET initiative's recommendation regarding the establishment of standards for outcome selection [42], this review will also appraise the inclusiveness of endpoint measurement over time. In addition to publications, we also plan to develop lay summaries that will be disseminated online and distributed to patient groups and key societies.

\section{Supplementary information}

Supplementary information accompanies this paper at https://doi.org/10. 1186/s13643-019-1195-1.

Additional file 1. PRISMA checklist.

Additional file 2. Search strategy.

Additional file 3. Data extraction form.

\section{Abbreviations}

MD: Meniere disease; NMA: Network meta-analysis; PICOs: Population, intervention, comparator, outcome, study design; PRESS: Peer review of electronic search strategies; PTA: Pure tone average; SDS: Speech discrimination score; SMD: Standardized mean difference; SR: Systematic review; SRT: Speech reception threshold; WRS: Word recognition score

\section{Acknowledgements}

We acknowledge Kaitryn Campbell for her assistance in peer-reviewing the search strategies for this planned systematic review.

\section{Authors' contributions}

$\mathrm{BH}, \mathrm{SK}$, and DW conceived the study design. $\mathrm{BH}, \mathrm{NA}$, and DW drafted the initial version of the protocol. BS derived the literature search strategy. All co-authors contributed to review of drafts of the manuscript. All authors approved the final submitted version of the manuscript. $\mathrm{BH}$ is the guarantor of the review. 


\section{Funding}

This systematic review and NMA was funded by Canadian Institutes of Health Research's (CIHR), Grant number PJT-153108. The funding agency was not involved in development of the protocol.

\section{Availability of data and materials \\ Not applicable}

\section{Ethics approval and consent to participate}

Not applicable

\section{Consent for publication}

Not applicable

\section{Competing interests}

$\mathrm{BH}$ has received consultancy fees from Cornerstone Research Group for methodologic advice related to systematic reviews and meta-analysis. The remaining authors declare that they have no competing interests.

\section{Author details}

${ }^{1}$ Center for Practice Changing Research, Ottawa Hospital Research Institute, 501 Smyth Road, Box 201B, Ottawa, ON K1H 8 L6, Canada. ${ }^{2}$ Department of Otolaryngology-Head and Neck Surgery, The Ottawa Hospital, 501 Smyth Rd, Ottawa, ON K1H 8L6, Canada. ${ }^{3}$ Faculty of Health Sciences, University of Ottawa, Ottawa, Canada. ${ }^{4}$ Children's Hospital of Eastern Ontario Research Institute, Ottawa, Canada. ${ }^{5}$ Department of Otolaryngology - Head \& Neck Surgery, Sunnybrook Research Institute, Sunnybrook Health Sciences Centre, Toronto, Canada. ${ }^{6}$ Faculty of Medicine, University of Toronto, Toronto, Canada. ${ }^{7}$ Medicine Prof. Corp, Ottawa, Canada. ${ }^{8}$ The University of Ottawa Faculty of Epidemiology and Community Medicine, 451 Smyth Rd, Ottawa, ON K1H 8L1, Canada.

\section{Received: 24 January 2019 Accepted: 13 October 2019}

\section{Published online: 30 December 2019}

\section{References}

1. Canadian Hearing Society. Facts and figures: prevalence of hearing loss. Available From: https://www.chs.ca/facts-and-figures. 2017.

2. Mathers C, Stein C, Ma Fat D, Rao C, Inoue M, Tomijima N, et al. Global burden of disease in the year 2000. Available from: http://www.who.int/ healthinfo/paper50.pdf. 2002

3. Harkonen K, Kivekas I, Rautiainen M, Kotti V, Sivonen V, Vasama JP. Singlesided deafness: the effect of cochlear implantation on quality of life, quality of hearing, and working performance. ORLJ Otorhinolaryngol Relat Spec. 2015;77:339-45.

4. Anderson JP, Harris JP. Impact of Meniere's disease on quality of life. Otol Neurotol. 2001;22(6):888-94.

5. Naude A. Clinical and audiological features of Meniere's disease: insight into the diagnostic process. 2006. Available from: https://repository.up.ac.za/ handle/2263/27851

6. Kirby SE, Yardley L. Cognitions associated with anxiety in Meniere's disease J Psychosom Res. 2009:(2):111-8.

7. Gurkov R, Pyyko I, Zou J, Kentala E. What is Meniere's disease? A contemporary re-evaluation of endolymphatic hydrops. J Neurol. 2016; 263(Suppl 1):S71-81.

8. Yokota Y, Kitahara T, Sakagami M, Ito T, Kimura T, Okayasu T, et al. Surgical results and psychological status in patients with intractable Meniere's disease. Auris Nasus Larynx. 2016;43(3):287-91.

9. Committee on Hearing and Equilibrium guidelines for the diagnosis and evaluation of therapy in Meniere's disease. American Academy of Otolaryngology-Head and Neck Foundation, Inc. Otolaryngol Head Neck Surg. 1995;113(3):181-5.

10. da Costa SS, de Sousa LC, Piza MR. Meniere's disease: overview, epidemiology, and natural history. Otolaryngol Clin North Am. 2002;35(3): 455-95

11. Syed MI, Ilan O, Leong AC, Pothier DD, Rutka JA. Meniere's syndrome or disease: time trends in management and quality of evidence over the last two decades. Otol Neurotol. 2015;36(8):1309-16.

12. Shamseer L, Moher D, Clarke M, Ghersi D, Liberati A, Petticrew M, et al. Preferred reporting items for systematic review and meta-analysis protocols (PRISMA-P) 2015: elaboration and explanation. BMJ. 2015;350:g7647.
13. National Institute for Health Research. PROSPERO: International prospective register of systematic reviews. Available from: https://www.crd.york.ac.uk/ prospero/. 2017

14. Hutton B, Salanti G, Caldwell DM, Chaimani A, Schmid CH, Cameron C, et al. The PRISMA extension statement for reporting of systematic reviews incorporating network meta-analyses of health care interventions: checklist and explanations. Ann Intern Med. 2015;162(11):777-84.

15. Acharya A, Singh MM, Shrestha A. First line treatment of Meniere's disease. J Lumbini Med Coll. 2016:4(2):68-71.

16. McGowan J, Sampson M, Salzwedel DM, Cogo E, Foerster V, Lefebvre C. PRESS Peer review of electronic search strategies: 2015 guideline statement. J Clin Epidemiol. 2016;75:40-6.

17. Khangura S, Konnyu K, Cushman R, Grimshaw J, Moher D. Evidence summaries: the evolution of a rapid review approach. Syst Rev. 2012;1:10.

18. Moher D, Liberati A, Tetzlaff J, Altman DG. Preferred reporting items for systematic reviews and meta-analyses: the PRISMA statement. PLoS Med. 2009;6(7):e1000097.

19. Core Outcome Measures in Effectiveness Trials Initiative (COMET). [cited 2019 Apr 11]. Available from: http://www.comet-initiative.org/studies/search

20. Development of a core outcome set for clinical trials in Menière's disease - a pilot survey of healthcare professionals and patients. :. Core Outcome Measures in Effectiveness Trials Initiative (COMET). [cited 2019 Apr 11]. Available from: http://www.comet-initiative.org/studies/details/818?result=true

21. Higgins JP, Altman DG, Gotzsche PC, Juni P, Moher D, Oxman AD, et al. The Cochrane Collaboration's tool for assessing risk of bias in randomised trials. BMJ. 2011;343:d5928.

22. Tyrrell JS, Whinney DJ, Ukoumunne OC, Fleming LE, Osborne NJ. Prevalence, associated factors, and comorbid conditions for Meniere's disease. Ear Hear. 2014;35(4):e162-9.

23. Mohamed S, Khan I, Iliodromiti S, Gaggini M, Kontorinis G. Meniere's disease and underlying medical and mental conditions: towards factors contributing to the disease. ORLJ Otorhinolaryngol Relat Spec. 2016:78(3): 144-50

24. Harcourt J, Barraclough K, Bronstein AM. Meniere's disease. BMJ. 2014;349: g6544.

25. Pieska T, Kotimaki J, Mannikko M, Sorri M, Hietikko E. Concomitant diseases and their effect on disease prognosis in Meniere's disease: diabetes mellitus identified as a negative prognostic factor. Acta Otolaryngol. 2018;138(1):36-4.

26. Dias S, Welton N, Sutton A, Ades A. NICE DSU technical support document 2: a generalised linear modelling framework for pairwise and network metaanalysis of randomised controlled trials. https://www.ncbi.n/m.nih.gov/ books/n/nicedsutsd2/pdf/. 2014.

27. Dias S, Sutton A, Welton N, Ades A. NICE DSU technical support document 3: heterogeneity: subgroups, meta-regression, bias and bias-adjustment. https://www.ncbi.n/m.nih.gov/pubmedhealth/n/nicetechsup3/pdf/. 2018

28. Dias S, Welton N, Sutton A, Caldwell DM, Lu G, Ades A. NICE DSU technical support document 4: inconsistency in networks of evidence based on randomised controlled trials. https://www.ncbi.nlm.nih.gov/pubmedhealth/ n/nicedsutsd4/pdf/. 2014.

29. Spiegelhalter DJ, Best NG, Carlin BP, Van Der Linde A. Bayesian measures of model complexity and fit. J R Stat Soc B. 2002;64(4):583-639.

30. Johnston BC, Thorlund K, Schunemann HJ, Xie F, Murad MH, Montori VM, et al. Improving the interpretation of quality of life evidence in metaanalyses: the application of minimal important difference units. Health Qual Life Outcomes. 2010:8:116.

31. Spiegelhalter D, Thomas A, Best N, Dave L. Manuals - OpenBUGS. OpenBUGS User Manual: Version 3.2.3. 2014. [cited 2018 Jul 19]. Available from: http://www.openbugs.net/w/Manuals

32. R2WinBUGS: A Package for Running WinBUGS from R | Sturtz | I Stat Software. [cited 2018 Jul 20]; Available from: https://www.jstatsoft.org/article/view/v012i03

33. Thorlund K, Walter SD, Johnston BC, Furukawa TA, Guyatt GH. Pooling health-related quality of life outcomes in meta-analysis-a tutorial and review of methods for enhancing interpretability. Res Synth Methods. 2011;2(3): 188-203.

34. Frejo L, Martin-Sanz E, Teggi R, Trinidad G, Soto-Varela A, Santos-Perez S, et al. Extended phenotype and clinical subgroups in unilateral Meniere disease: a cross-sectional study with cluster analysis. Clin Otolaryngol. 42(6): 1172-80.

35. Syed MI, Rutka JA, Hendry J, Browning GG. Positive pressure therapy for Meniere's syndrome/disease with a Meniett device: a systematic review of randomised controlled trials. Clin Otolaryngol. 2015;40(3):197-207. 
36. Sood AJ, Lambert PR, Nguyen SA, Meyer TA. Endolymphatic sac surgery for Meniere's disease: a systematic review and meta-analysis. Otol Neurotol. 2014;35(6):1033-45.

37. Lim MY, Zhang M, Yuen HW, Leong JL. Current evidence for endolymphatic sac surgery in the treatment of Meniere's disease: a systematic review. Singap Med J. 2015;56(11):593-8.

38. Crowson MG, Patki A, Tucci DL. A systematic Review of diuretics in the medical management of Meniere's disease. Otolaryngol Head Neck Surg. 2016;154(5):824-34.

39. Salanti G. Indirect and mixed-treatment comparison, network, or multipletreatments meta-analysis: many names, many benefits, many concerns for the next generation evidence synthesis tool. Res Synth Methods. 2012;3(2): 80-97.

40. Caldwell DM, Ades AE, Higgins JP. Simultaneous comparison of multiple treatments: combining direct and indirect evidence. BMJ. 2005:331(7521): 897-900.

41. Catala-Lopez F, Tobias A, Cameron C, Moher D, Hutton B. Network metaanalysis for comparing treatment effects of multiple interventions: an introduction. Rheumatol Int. 2014;34(11):1489-96.

42. Kirkham JJ, Davis K, Altman DG, Blazeby JM, Clarke M, Tunis S, et al. Core outcome set-standards for development: the COS-STAD recommendations. PLOS Med. 2017:14(11):e1002447.

\section{Publisher's Note}

Springer Nature remains neutral with regard to jurisdictional claims in published maps and institutional affiliations.

Ready to submit your research? Choose BMC and benefit from:

- fast, convenient online submission

- thorough peer review by experienced researchers in your field

- rapid publication on acceptance

- support for research data, including large and complex data types

- gold Open Access which fosters wider collaboration and increased citations

- maximum visibility for your research: over $100 \mathrm{M}$ website views per year

At BMC, research is always in progress.

Learn more biomedcentral.com/submissions 